\title{
On Schrödinger's bridge problem
}

\author{
Shmuel Friedland*
}

September 3, 2016

\begin{abstract}
In the first part of this paper we generalize the result of Georgiou-Pavon that a positive square matrix can be scaled uniquely to a column stochastic matrix which maps a given positive probability vector to another given positive probability vector. In the second part of this paper we prove that a positive quantum channel can be scaled to another positive quantum channel which maps a given positive definite density matrix to another given positive definite density matrix using Brower's fixed point theorem. This result proves the Georgiou-Pavon conjecture for two positive definite density matrices in their recent paper [6]. We show uniqueness of fixed points for certain two positive definite density matrices.
\end{abstract}

Keywords: scaling of matrices, scaling of quantum channels, Schrödinger's bridge problem, fixed points.

2010 Mathematics Subject Classification. 15B51, 15B57, 32A05, 55M20, $81 \mathrm{P} 45$.

\section{Introduction}

The classical Schrödinger bridge problem, studied by Schrödinger in [12, 13], seeks the most likely probability law for a diffusion process, in path space, that matches marginals at two end points in time. The discrete version of Schrödinger's bridge problem for Markov chains, as I understand, can be stated succinctly as follows. Let $A$ be an $n \times n$ a stochastic matrix. That is, $A$ is a nonnegative matrix, and $A^{\top} \mathbf{1}=\mathbf{1}, \mathbf{1}=(1, \ldots, 1)^{\top}$. (Sometimes $A$ is called column stochastic.) Let $\mathbf{a}, \mathbf{b}$ be two positive (column) probability vectors. Does there exists a scaling of $A$, i.e., $B=D_{1} A D_{2}$, where $D_{1}, D_{2}$ are two $n \times n$ diagonal matrices with positive diagonal entries, such that $B$ is stochastic and $B \mathbf{a}=\mathbf{b}$ ? See [6] for the motivation of this problem and $\S 2$.

For $\mathbf{a}=\mathbf{b}=\mathbf{1}$ this problem is equivalent to the well known problem: when a nonnegative $A \in \mathbb{R}^{n \times n}, A \geq 0$, can be scaled to a doubly stochastic matrix? This problem was answered by Sinkhorn [14]. Namely, it is always possible to scale a matrix $A$ to a doubly stochastic if all the entries of $A$ are positive, i.e. $A>0$. In that case $D_{1}, D_{2}$ are unique (up to : $t D_{1}, t^{-1} D_{2}$ for $t>0$ ). The unique scaling of a fully indecomposable $A \geq 0$ to a doubly stochastic matrix was proven in [11, 3, 15]. ( $A \geq 0$ fully indecomposable if $P A Q$ is irreducible for any pair of permutation

\footnotetext{
* Department of Mathematics, Statistics and Computer Science, University of Illinois at Chicago, Chicago, Illinois 60607-7045, USA, friedlan@uic.edu.
} 
matrices $P, Q$.) Necessary and sufficient conditions for case $\mathbf{a}=\mathbf{1}$ was given in $[2,9]$. Theorem 3.2 in [5] proves the existence of unique scaling of a fully indecomposable $A$ for $\mathbf{a}=\mathbf{b}$.

In a recent paper Georgiou and Pavon [6] proved that Schrödinger's bridge problem is uniquely solvable for $A>0$. Their proof is based on the strict contraction of a corresponding map with respect to the Hilbert metric. In the first part of this paper we give a different proof of this result. We also give a generalization of Schrödinger's bridge problem to a nonnegative $A$ with no zero column and $A A^{\top}$ irreducible. Georgiou and Pavon considered in [6] an analog of Schrödinger 's bridge problem for quantum channels. The simplified version of this problem is: Denote by $\mathbb{C}^{n \times n}$ the set of $n \times n$ complex valued matrices, and by $\mathrm{H}_{n} \supset \mathrm{H}_{n,+} \supset \mathrm{H}_{n,++}$ the subsets of hermitian, positive semi-definite, and positive definite matrices, respectively, in $\mathbb{C}^{n \times n}$. Let $\mathrm{H}_{n,+, 1} \supset \mathrm{H}_{n,++, 1}$ be the subset positive semi-definite and positive definite matrices of trace 1 , respectively, in $\mathrm{H}_{n,+}$. Let $\mathbf{G L}(n, \mathbb{C}) \subset \mathbb{C}^{n \times n}$ be the group of invertible matrices. Recall that $Q: \mathbb{C}^{n \times n} \rightarrow \mathbb{C}^{n \times n}$ is called a completely positive operator if

$$
Q(X)=\sum_{i=1}^{k} A_{i} X A_{i}^{*}, \quad A_{i} \in \mathbb{C}^{n \times n}, i=1, \ldots, k, \quad X \in \mathbb{C}^{n \times n} .
$$

Assume that $Q$ is completely positive. $Q$ is called positive if $Q\left(\mathrm{H}_{n,+, 1}\right) \subset \mathrm{H}_{n,++} \cdot Q$ is called quantum channel if

$$
\sum_{i=1}^{k} A_{i}^{*} A_{i}=I_{n}
$$

Note that a quantum channel is trace preserving, which is equivalent to $Q\left(\mathrm{H}_{n,+, 1}\right) \subseteq$ $\mathrm{H}_{n,+, 1} \cdot R: \mathbb{C}^{n \times n} \rightarrow \mathbb{C}^{n \times n}$ is called a scaling of $Q$ if

$$
R(X)=S Q\left(T X T^{*}\right) S^{*}, \text { for fixed } S, T \in \mathbf{G L}(n, \mathbb{C}) \text { and all } X \in \mathbb{C}^{n \times n} .
$$

The simplified version Schrödinger's bridge problem for quantum channels is:

Problem 1.1 Let $Q: \mathbb{C}^{n \times n} \rightarrow \mathbb{C}^{n \times n}$ be a positive quantum channel. Assume that $\alpha, \beta \in \mathrm{H}_{n,++, 1}$. Does there exists a scaling of $Q$ to a quantum channel $R$ that satisfies $R(\alpha)=\beta$.

Problem 1.1 for $\alpha=\beta=\frac{1}{n} I_{n}$ was solved by Gurvits [7] and in [6] by different methods. (This is the analog of Sinkhorn's theorem.) Conjecture 1 in [6] implies the solution of Problem 1.1. In this paper we show that Problem 1.1 is solvable using Brouwer's fixed point theorem, similar to the methods in [6]. This result yields a solution of the Georgiou-Pavon conjecture for two positive definite density matrices. We also show that for $\alpha, \beta$ in some neighborhood of $\frac{1}{n} I_{n}$, depending on $Q$, the scaling of $Q$ is "unique" in certain sense. That is, the corresponding map has a unique fixed point.

We now summarize briefly the contents of this paper. In $\S 2$ we discuss Schrödinger's bridge problem for stochastic matrices and its generalization to certain nonnegative matrices. $\S 3$ discusses some known results on quantum channels that are used in the next sections. In $\S 4$ give a solution to Problem 1.1 using Brower's fixed point theorem. We also show that a solution to Problem 1.1 is equivalent to $[6$, Conjecture 
1] for two positive definite density matrices. In $\S 5$ we show that the map constructed to solve Problem 1.1 has a unique fixed point if the two density matrices are in the neighborhood of the uniform density matrix.

\section{Schrödinger's bridge problem for stochastic matrices}

Denote $\mathbb{R}_{+}=[0, \infty), \mathbb{R}_{++}=(0, \infty)$. For column vectors $\mathbf{u}=\left(u_{1}, \ldots, u_{n}\right)^{\top}, \mathbf{v}=$ $\left(v_{1}, \ldots, v_{n}\right)^{\top}$ let $\mathbf{u} \circ \mathbf{v}=\left(u_{1} v_{1}, \ldots, u_{n} v_{n}\right)^{\top}, D(\mathbf{u})=\operatorname{diag}\left(u_{1}, \ldots, u_{n}\right) \in \mathbb{R}^{n \times n}$.

Schrödinger's bridge problem is stated as follows [6]. Let $A=\left[a_{i j}\right] \in \mathbb{R}_{++}^{n \times n}$, and let two probability vectors $\mathbf{a}, \mathbf{b} \in \mathbb{R}_{++}^{n}$ be given. Do there exist $\mathbf{u}, \mathbf{v}, \mathbf{x}, \mathbf{y} \in \mathbb{R}_{++}^{n}$ such that

$$
\mathbf{v}=A^{\top} \mathbf{u}, \quad \mathbf{y}=A \mathbf{x}, \quad \mathbf{a}=\mathbf{v} \circ \mathbf{x}, \quad \mathbf{b}=\mathbf{u} \circ \mathbf{y} .
$$

A straightforward calculation yields that if (2.1) holds then the matrix $B=D(\mathbf{u}) A D(\mathbf{v})^{-1}$ satisfies:

$$
B^{\top} \mathbf{1}=\mathbf{1}, B \mathbf{a}=\mathbf{b}, \quad B \in \mathbb{R}_{++}^{n \times n}, \mathbf{a}, \mathbf{b} \in \mathbb{R}_{++}^{n}, \mathbf{1}^{\top} \mathbf{a}=\mathbf{1}^{\top} \mathbf{b}=1 .
$$

Vice versa, if $B=D(\mathbf{u}) A D(\mathbf{v})^{-1}$ satisfies the above equation then (2.1) holds, where $\mathbf{x}, \mathbf{y}$ are determined uniquely by the last two conditions of (2.1).

Note that any $A \in \mathbb{R}_{++}^{n \times n}$ is uniquely scaled from the right to a stochastic matrix. That is there exists a unique $D_{2}=D\left(A^{\top} \mathbf{1}\right)^{-1}$ such that $A D_{2}$ is stochastic. Hence without loss of generality we can assume that $A \in \mathbb{R}_{++}^{n \times n}$ is stochastic.

It is shown in [5, Theorem 3.2], that for given fully indecomposable $A \in \mathbb{R}_{+}^{n \times n}$ and $\mathbf{u}, \mathbf{v} \in \mathbb{R}_{++}^{n}$ there exists a unique scaling of $A$ such that $D_{1} A D_{2} \mathbf{u}=\mathbf{u}$ and $D_{2} A^{\top} D_{1} \mathbf{v}=\mathbf{v}$. Choose $\mathbf{a}=\mathbf{b}=\mathbf{u}$ and $\mathbf{v}=\mathbf{1}$ to deduce the solution of Schrödinger's bridge problem in this case. It is straightforward to show that a solution of Schrödinger's bridge problem for $\mathbf{a}=\mathbf{b}$ implies the above cited result in [5] by considering the matrix $D(\mathbf{v})^{-1} D_{1} A D_{2} D(\mathbf{v})$ and $\mathbf{a}=\mathbf{b}=\mathbf{u} \circ \mathbf{v}$.

Denote $\mathbb{P R}_{++}^{n}$ the projective space associated with the open cone of positive vectors in $\mathbb{R}^{n}$. That is, $\mathbb{P R}_{++}^{n}$ is the set of open rays $R(\mathbf{u})=\{t \mathbf{u}, t>0, \mathbf{u} \in$ $\left.\mathbb{R}_{++}^{n}\right\}$. Denote by $\Pi_{n} \subset \mathbb{R}^{n}$ the simplex of probability vectors. Clearly, $\mathbb{P R}_{++}^{n}$ is isomorphic to the interior of $\Pi_{n}$, denoted as $\Pi_{n,++}=\Pi_{n} \cap \mathbb{R}_{++}^{n}$. A one point compactification of $\Pi_{n,++}$ is the identification of the points $\partial \Pi_{n,++}$, the boundary of $\Pi_{n,++} \subset \mathbb{R}^{n}$, to one point, denoted as $\infty$. Denote by $\widehat{\Pi_{n}}=\Pi_{n,++} \cup\{\infty\}$ the above one point compactification of $\Pi_{n}$. It is well known that $\widehat{\Pi}_{n}$ is homeomorphic to the $n-1$ dimensional sphere $\mathrm{S}^{n-1}=\left\{\mathbf{x} \in \mathbb{R}^{n}, \mathbf{x}^{\top} \mathbf{x}=1\right\}$. Moreover, $\mathrm{S}^{n-1}$ can be viewed as one point compactification of $\mathbb{R}^{n-1}: \widehat{\mathbb{R}^{n-1}}=\mathbb{R}^{n-1} \cup\{\infty\}$. Similarly, one point compactification of $\mathbb{P R}_{++}^{n}, \widehat{\mathbb{P R}_{++}^{n}}=\mathbb{P R}_{++}^{n} \cup\{\infty\}$, is homeomorphic to $\mathrm{S}^{n-1}$, equivalently homeomorphic to $\widehat{\Pi_{n}}$.

Denote by $\Sigma_{n} \subset \mathbb{R}_{+}^{n \times n}$ the convex set of stochastic matrices. Let $\Sigma_{n,++}=$ $\Sigma_{n} \cap \mathbb{R}_{++}^{n \times n}$ be the interior of $\Sigma_{n}$.

Assume that $A \in \mathbb{R}_{+}^{n \times n}$ is a matrix with no zero column. Let

$$
\Phi_{A}: \mathbb{R}_{++}^{n} \rightarrow \Sigma_{n}, \quad \Phi_{A}(\mathbf{x})=D(\mathbf{x}) A D\left(A^{\top} \mathbf{x}\right)^{-1} \text { for } \mathbf{x} \in \mathbb{R}_{++}^{n} .
$$

Clearly, for each $t>0$ and $\mathbf{x} \in \mathbb{R}_{++}^{n}$ we have that $\Phi_{A}(t \mathbf{x})=\Phi_{A}(\mathbf{x})$. Hence can be viewed as smooth map $\tilde{\Phi}_{A}: \mathbb{P R}_{++}^{n} \rightarrow \Sigma_{n}$. Clearly, $\tilde{\Phi}_{A}$ can be viewed also as the restriction of $\Phi_{A}$ to $\Pi_{n,++}$. Note that if $A>0$ then $\Phi_{A}\left(\mathbb{R}_{++}^{n}\right) \subset \Sigma_{n,++}$. 
The following theorem generalizes [6, Theorem 3].

Theorem 2.1 Let $A \in \mathbb{R}_{+}^{n \times n}$ and $\mathbf{a} \in \Pi_{n,++}$ be given. Assume that $A$ does not have a zero column or zero row. Consider the map

$$
\Phi_{A, \mathbf{a}}: \Pi_{n,++} \rightarrow \Pi_{n,++}, \quad \Phi_{A, \mathbf{a}}(\mathbf{x})=\Phi_{A}(\mathbf{x}) \mathbf{a} \text { for } \mathbf{x} \in \Pi_{n,++} .
$$

1. Assume that $A$ is a positive matrix. Then the map $\Phi_{A, \mathbf{a}}$ extends to a continuous map of $\Pi_{n}$. It maps the boundary of $\Pi_{n}$ to its boundary. Furthermore, $\Phi_{A, \mathbf{a}}$ is a self-diffeomorphism of $\Pi_{n,++}$.

2. Assume that $A A^{\top}$ is an irreducible matrix. Then $\Phi_{A, \mathbf{a}}$ is a diffeomorphism of $\Pi_{n,++}$ and $\Phi_{A, \mathbf{a}}\left(\Pi_{n,++}\right)$.

Proof. As $A$ is a nonnegative matrix with no zero column the map (2.4) is a well defined smooth map. As $A$ does not have a zero row, $\Phi_{A}(\mathbf{x})$ does not have zero row for $\mathbf{x} \in \Pi_{n,++}$. Therefore $\Phi_{A}(\mathbf{x}) \mathbf{a} \in \Pi_{n,++}$. Hence $\Phi_{A, \mathbf{a}}$ is a self-smooth map of $\Pi_{n,++}$, i.e. $\Phi_{A, \mathbf{a}}\left(\Phi_{n,++}\right) \subseteq \Pi_{n,++}$.

1. Assume that $A>0$. Then $\Phi_{A}: \Pi_{n} \rightarrow \Sigma_{n}$ is continuous. In particular, $\Phi_{A}(\mathbf{x}) \mathbf{a} \in$ $\Pi_{n}$ for each $\mathbf{x} \in \Pi_{n}$. Therefore $\Phi_{A, \mathbf{a}}$ is a continuous map of $\Pi_{n}$ to itself. Let $\mathbf{x}=\left(x_{1}, \ldots, x_{n}\right)^{\top} \in \partial \Pi_{n}$. Thus $x_{i}=0$ for some $i$. Hence the $i-t h$ row of $\Phi_{A}(\mathbf{x})$ is zero. Therefore the $i$-th coordinate of $\Phi_{A}(\mathbf{x}) \mathbf{a}$ is zero. Thus $\Phi_{A}(\mathbf{x}) \mathbf{a} \in \partial \Pi_{n,++}$. Hence $\Phi_{A, \mathbf{a}}\left(\partial \Pi_{n,++}\right) \subseteq \partial \Pi_{n,++}$. These results yield that $\Phi_{A, a}: \Pi_{n} \rightarrow \Pi_{n}$ induces a continuous map $\widehat{\Phi_{A, \mathbf{a}}}: \widehat{\Pi_{n}} \rightarrow \widehat{\Pi_{n}}$. More precisely the map $\Phi_{A, \mathbf{a}}: \Pi_{n,++} \rightarrow \Pi_{n,++}$ is a proper map. That is, given a sequence $\mathbf{x}_{m} \in \Pi_{n,++}, m \in \mathbb{N}$ which converges to $\partial \Pi_{n,++}$ then the sequence $\Phi_{A, \mathbf{a}}\left(\mathbf{x}_{m}\right), m \in \mathbb{N}$ converges to $\partial \Pi_{n,++}$.

We now show that $\Phi_{A, \mathbf{a}}: \Pi_{n,++} \rightarrow \Pi_{n,++}$ is a local diffeomorphism. Assume that $\mathbf{x} \in \Pi_{n,++}$. Then the neighborhood of $\mathbf{x}$ in $\Pi_{n}$ are points of the form $\mathbf{x}+t \mathbf{w}, \mathbf{w}^{\top} \mathbf{w}=$ $1, \mathbf{1}^{\top} \mathbf{W}=0,|t|<\varepsilon$ for some small $\varepsilon>0$. Equivalently, let $\mathbf{W} \subset \mathbb{R}^{n}$ be the subspace of all vectors orthogonal to $\mathbf{1}$. Then the neighborhood of $\mathbf{x}$ is diffeomorphic to an open ball of radius $\varepsilon>0$ centered at $\mathbf{0}$ in $\mathbf{W}$.

Suppose first that $A$ is stochastic. Assume that $\mathbf{x}=\frac{1}{n} \mathbf{1}$. Thus

$$
\begin{aligned}
& A^{\top}(\mathbf{x}+t \mathbf{w})=\mathbf{x}+t A^{\top} \mathbf{w},\left(A^{\top}(\mathbf{x}+t \mathbf{w})\right)_{i}^{-1}=n\left(\mathbf{1}-n t A^{\top} \mathbf{w}\right)+O\left(t^{2}\right) \\
& D\left(A^{\top}(\mathbf{x}+t \mathbf{w})\right)^{-1}=n\left(I-n t D\left(A^{\top} \mathbf{w}\right)\right)+O\left(t^{2}\right) \\
& \Phi_{A}(\mathbf{x}+t \mathbf{w})=A+n t\left(D(\mathbf{w}) A-A D\left(A^{\top} \mathbf{w}\right)\right)+O\left(t^{2}\right) .
\end{aligned}
$$

Assume that $A \mathbf{a}=\mathbf{b}$. Then

$\Phi_{A, \mathbf{a}}(\mathbf{x}+t \mathbf{w})=\mathbf{b}+n t\left(D(\mathbf{w}) \mathbf{b}-A D\left(A^{\top} \mathbf{w}\right) \mathbf{a}\right)+O\left(t^{2}\right)=\mathbf{b}+n t\left(D(\mathbf{b})-A D(\mathbf{a}) A^{\top}\right) \mathbf{w}+O\left(t^{2}\right)$.

Let

$$
F(A, \mathbf{a})=D(\mathbf{b})-A D(\mathbf{a}) A^{\top}=D(A \mathbf{a})-A D(\mathbf{a}) A^{\top} .
$$

Clearly $F(A, \mathbf{a})$ is symmetric matrix satisfying

$$
F(A, \mathbf{a}) \mathbf{1}=D(\mathbf{b}) \mathbf{1}-A D(\mathbf{a}) A^{\top} \mathbf{1}=\mathbf{b}-A D(\mathbf{a}) \mathbf{1}=\mathbf{b}-A \mathbf{a}=\mathbf{0} .
$$

Hence $\mathbf{W}$ is an invariant subspace of $F(A, \mathbf{a})$. Furthermore, $n F(A, \mathbf{a}) \mid \mathbf{W}$ is the Jacobian of $\Phi_{A, \mathbf{a}}$ at $\mathbf{x}=\frac{1}{n}$. 
We claim that the $n-1$ eigenvalues of $F(A, \mathbf{a}) \mid \mathbf{W}$ are positive. This claim follows from the observation that $F(A, \mathbf{a})$ a symmetric irreducible singular M-matrix [4, $\S 6.6]$. Indeed, the matrix $r I-F(A, \mathbf{a})>0$ for $r \gg 1$. Clearly $(r I-F(A, \mathbf{a})) \mathbf{1}=r \mathbf{1}$. Hence $r$ is the spectral radius of $(r I-F(A, \mathbf{a})), r$ is a simple eigenvalue of $r I-F(A, \mathbf{a})$ and all other eigenvalues of $r I-F(A, \mathbf{a})$ are strictly less than $r$. Hence $-F(A, \mathbf{a})$ is a singular symmetric matrix, which has $n-1$ negative eigenvalues. In particular $F(A, \mathbf{a}) \mid \mathbf{W}$ is an invertible transformation. Hence $\Phi_{A, \mathbf{a}}$ is a local diffeomorphism at $\mathbf{x}=\frac{1}{n} \mathbf{1}$. For a general $\mathbf{x} \in \Pi_{n,++}$ if follows that the Jacobian of $\Phi_{A, \mathbf{a}}$ at $\mathbf{x}$ is $n F\left(\Phi_{A}(\mathbf{x}), \mathbf{a}\right) \mid \mathbf{W}$. Hence $\Phi_{A, a}$ is a local diffeomorphism on $\Pi_{n,++}$.

As $\Phi_{A, \mathbf{a}}: \Pi_{n,++} \rightarrow \Pi_{n,++}$ is a proper map and a local diffeomorphism it follows that $\Phi_{A, \mathbf{a}}$ is proper cover of $\Pi_{n,++}$ by $\Pi_{n,++}$. As $\Pi_{n,++}$ is simply connected we deduce that $\Phi_{A, \mathbf{a}}$ is a diffeomorphism of $\Pi_{n,++} \cdot$ (One can also prove this fact by using the degree theory [10].)

2. It is left to discuss the theorem where $A$ is a nonnegative matrix with some zero entries, with no zero columns and $A A^{\top}$ is irreducible. (This condition yields that $A$ has no zero rows.) We claim first that $\Phi_{A, \mathbf{a}}$ is a local diffeomorphism. As above, the Jacobian of $\Phi_{A, \mathbf{a}}$ at $\mathbf{x} \in \Pi_{n,++}$ is $n$ times the restriction of

$$
F\left(\Phi_{A}(\mathbf{x}), \mathbf{a}\right)=D\left(\Phi_{A}(\mathbf{x}) \mathbf{a}\right)-\Phi_{A}(\mathbf{x}) D(\mathbf{a}) \Phi_{A}(\mathbf{x})^{\top}
$$

to W. Clearly, $F\left(\Phi_{A}(\mathbf{x}), \mathbf{a}\right) \mathbf{1}=\mathbf{0}$. We claim that $F\left(\Phi_{A}(\mathbf{x}), \mathbf{a}\right)$ is an irreducible singular M-matrix. Indeed, $(i, j)$ off-diagonal entry of $-F\left(\Phi_{A}(\mathbf{x}), \mathbf{a}\right)$ is positive if and only if the $(i, j)$ entry of $A A^{\top}$ is positive. Since $A A^{\top}$ is an irreducible matrix it follows that $F\left(\Phi_{A}(\mathbf{x}), \mathbf{a}\right)$ a symmetric irreducible singular $M$-matrix. Hence the eigenvalues of $F\left(\Phi_{A}(\mathbf{x}), \mathbf{a}\right) \mid \mathbf{W}$ are positive, and the Jacobian of $\Phi_{A, \mathbf{a}}$ at $\mathbf{x}$ is invertible.

It is left to show that $\Phi_{A, \mathbf{a}}: \Pi_{n,++} \rightarrow \Phi_{A, \mathbf{a}}\left(\Pi_{n,++}\right)$ is one-to-one. Assume to the contrary that there exists $\mathbf{x}_{1}, \mathbf{x}_{2} \in \Pi_{n,++}, \mathbf{x}_{1} \neq \mathbf{x}_{2}$ such that $\mathbf{b}=\Phi_{A, \mathbf{a}}\left(\mathbf{x}_{1}\right)=$ $\Phi_{A, \mathbf{a}}\left(\mathbf{x}_{2}\right)$. Since $\Phi_{A, \mathbf{a}}$ is a local diffeomorphism the following conditions hold. There exists a closed ball of a small radius $\varepsilon$ centered at $\mathbf{0}: B(\mathbf{0}, \varepsilon) \subset \mathbf{W}$, such that

$$
\mathbf{x}_{1}+B(\mathbf{0}, \varepsilon), \mathbf{x}_{2}+B(\mathbf{0}, \varepsilon) \subset \mathbb{P}_{n,++}, \quad\left(\mathbf{x}_{1}+B(\mathbf{0}, \varepsilon)\right) \cap\left(\mathbf{x}_{2}+B(\mathbf{0}, \varepsilon)\right)=\emptyset,
$$

and there exists $\varepsilon^{\prime}>0$ such that

$$
\Phi_{A, \mathbf{a}}\left(\mathbf{x}_{1}+B(\mathbf{0}, \varepsilon)\right) \cap \Phi_{A, \mathbf{a}}\left(\mathbf{x}_{2}+B(\mathbf{0}, \varepsilon)\right) \supset \mathbf{b}+B\left(\mathbf{0}, \varepsilon^{\prime}\right) .
$$

Assume that $t>0$ is small and let $A(t)=A+t \mathbf{1 1}^{\top}>0$. So $A(t)$ is a positive perturbation of $A$. Therefore $\Phi_{A(t), \mathbf{a}}$ is a perturbation of the map $\Phi_{A, \mathbf{a}}$ on the closed sets $\mathbf{x}_{1}+B(\mathbf{0}, \varepsilon), \mathbf{x}_{2}+B(\mathbf{0}, \varepsilon)$. The above condition yields that

$$
\Phi_{A(t), \mathbf{a}}\left(\mathbf{x}_{1}+B(\mathbf{0}, \varepsilon)\right) \cap \Phi_{A(t), \mathbf{a}}\left(\mathbf{x}_{2}+B(\mathbf{0}, \varepsilon)\right) \supset \mathbf{b}+B\left(\mathbf{0}, \varepsilon_{1}\right)
$$

for some $\varepsilon_{1} \in\left(0, \varepsilon^{\prime}\right)$ for some very small and positive $t$. This contradicts that $\Phi_{A(t), \mathbf{a}}$ is a self-diffeomorphism of $\Pi_{n,++}$.

We now discuss briefly the results of Theorem 2.1. Clearly, part 1 of this theorem is equivalent to the result of Georgiou-Pavon for positive matrices [6]. We now consider part 2 of this theorem. First note part 2 fails if $A A^{\top}$ is not irreducible. 
Indeed, suppose that $A$ is a permutation matrix. Then $\Phi_{A}(\mathbf{x})=A$ for each $\mathbf{x} \in$ $\Pi_{n,++}$. Therefore the map $\Phi_{A, \mathbf{a}}$ is a constant map. Note that $A A^{\top}=I$.

Suppose that $A$ is fully indecomposable. This is equivalent to the statement that $A=P B Q$, where $P$ and $Q$ are permutation matrices, $B=D+C$, such that $D$ is a diagonal matrix with positive diagonal entries and $C \geq 0$ is an irreducible matrix [3]. Then $A A^{\top}=P\left(D^{2}+D C^{\top}+C D+C C^{\top}\right) P^{\top}$ is irreducible. It is easy to given an example of a fully indecomposable $A$ such that $\partial \Phi_{A, \mathbf{a}}\left(\Pi_{n,++}\right) \cap \Pi_{n,++} \neq \emptyset$.

Assume that

$$
A=\left[\begin{array}{lll}
1 & 1 & 0 \\
0 & 1 & 1 \\
1 & 0 & 1
\end{array}\right], \mathbf{x}(t)=\left[\begin{array}{c}
t c_{1} \\
t c_{2} \\
1-t c_{1}-t c_{2}
\end{array}\right], c_{1}, c_{2}, t>0, c_{1}+c_{2}=1
$$

and $t \searrow 0$. Then

$$
\lim _{t \searrow 0} \Phi_{A}(\mathbf{x}(t))=B=\left[\begin{array}{ccc}
0 & c_{1} & 0 \\
0 & c_{2} & 0 \\
1 & 0 & 1
\end{array}\right], \quad \lim _{t \searrow 0} \Phi_{A, \mathbf{a}}(\mathbf{x}(t))=B \mathbf{a} .
$$

Hence $\mathbf{0}<B \mathbf{a} \in \partial \Phi_{A, \mathbf{a}}\left(\Pi_{3,++}\right)$.

Theorem 3.2 in [5] is equivalent to the statement that for a fully indecomposable $A$ and $\mathbf{a} \in \Pi_{n,++}$ one has $\mathbf{a} \in \Phi_{A, \mathbf{a}}\left(\Pi_{n,++}\right)$.

The results in $[2,9]$ can be stated as follows. Given a fully indecomposable matrix $A=\left[a_{i j}\right] \in \mathbb{R}_{+}^{n \times n}$ and $\mathbf{b}, \mathbf{c} \in \Pi_{n,++}$ there exist two diagonal matrices $D_{1}, D_{2} \in \mathbb{R}_{+}^{n \times n}$, with positive diagonal entries, such that $D_{1} A D_{2} \mathbf{1}=n \mathbf{b}, D_{2} A^{\top} D_{1} \mathbf{1}=n \mathbf{c}$ if and only if the following condition holds. There exists a matrix $C=\left[c_{i j}\right] \in \mathbb{R}_{+}^{n \times n}$ having the same pattern, i.e. $a_{i j}>0 \Longleftrightarrow c_{i j}>0$ for $i, j=1, \ldots, n$, such that $C \mathbf{1}=n \mathbf{b}, C^{\top} \mathbf{1}=n \mathbf{c}$. Hence for a fully indecomposable matrix $A$ one has $\mathbf{b} \in \Phi_{A, \frac{1}{n} \mathbf{1}}\left(\Pi_{n,++}\right)$ if and only if there exists a stochastic $C \in \mathbb{R}_{+}^{n \times n}$ having the same pattern as $A$ such that $C \mathbf{1}=n \mathbf{b}$.

Let $A$ and $B$ be defined as in (2.6) and (2.7) respectively. Clearly, $A$ is fully indecomposable. Let $\mathbf{b}=B\left(\frac{1}{3} \mathbf{1}\right)=\left(\frac{c_{1}}{3}, \frac{c_{2}}{3}, \frac{2}{3}\right)^{\top}$. It is straightforward to show that there is no stochastic matrix $C \in \mathbb{R}_{+}^{3 \times 3}$ with the same pattern as $A$ such that $C \mathbf{1}=3 \mathbf{b}$. So $\mathbf{b} \notin \Phi_{A, \frac{1}{3} \mathbf{1}}\left(\Pi_{3,++}\right)$ as we claimed.

\section{Preliminary results on quantum channels}

For $X \in \mathrm{H}_{n}$ arrange the eigenvalues of $X$ in a non increasing order $\lambda_{1}(X) \geq \cdots \geq$ $\lambda_{n}(X)$. Let trace $X$ be the trace of $\mathrm{X}: \operatorname{tr} X=\sum_{i=1}^{n} \lambda_{i}(X)$. Denote by $\|X\|_{F}=$ $\sqrt{\operatorname{tr} F^{2}}=\sqrt{\sum_{i=1}^{n} \lambda_{i}(X)^{2}}$ the Frobenius norm of $X$. For two real number $a \leq b$ denote by

$$
\mathrm{H}_{n}(a, b)=\left\{X \in \mathrm{H}_{n}, a \leq \lambda_{n}(X), \lambda_{1}(X) \leq b\right\} .
$$

It is well known that $\mathrm{H}_{n}(a, b)$ is a convex set. (Recall that $\lambda_{1}(X)$ and $-\lambda_{n}(X)$ are convex functions on $\mathrm{H}_{n}$ [4].) Let

$$
\mathrm{H}_{n}(a, b, 1):=\left\{X \in \mathrm{H}_{n}(a, b), \operatorname{tr} X=1\right\} .
$$

Note that $\mathrm{H}_{n}(a, b, 1) \neq \emptyset$ if and only if $a \leq \frac{1}{n} \leq b$. Furthermore, given $a \leq \frac{1}{n}$ then for each $X \in \mathrm{H}_{n}(a, b, 1)$ it follows that $\lambda_{1}(X) \leq 1-(n-1) a$. Clearly, for

$$
0<a \leq \frac{1}{n} \leq b \leq 1-(n-1) a
$$


the convex set $\mathrm{H}_{n}(a, b, 1)$ is a convex compact set of $\mathrm{H}_{n,++, 1}$. For a completely positive $Q$ let

$$
a(Q)=\min _{X \in \mathrm{H}_{n,+, 1}} \lambda_{n}(Q(X)), \quad b(Q)=\max _{X \in \mathrm{H}_{n,+, 1}} \lambda_{1}(Q(X)) .
$$

Clearly, $Q\left(\mathrm{H}_{n,+, 1}\right) \subseteq \mathrm{H}_{n}(a(Q), b(Q))$. Thus $Q$ is positive if and only if $a(Q)>0$. For a positive quantum channel $Q$ the constants $a(Q)$ and $b(Q)$ satisfy (3.2) and $Q\left(\mathrm{H}_{n,+, 1}\right) \subseteq \mathrm{H}_{n}(a(Q), b(Q), 1)$.

Let $\|X\|=\max _{\|\mathbf{x}\|=1}\|X \mathbf{x}\|$ be the spectral norm of $X \in \mathbb{C}^{n \times n}$. Recall that

$$
\|X\|=\lambda_{1}(X), \lambda_{n}(X)=\frac{1}{\lambda_{1}\left(X^{-1}\right)}=\frac{1}{\left\|X^{-1}\right\|}, \text { for } X \in \mathrm{H}_{n,++} .
$$

Furthermore, for each $X \in \mathrm{H}_{n,+}$ there exists a unique positive semi-definite matrix $X^{\frac{1}{2}} \in \mathrm{H}_{n,+}$, the square root of $X$, so that $\left(X^{\frac{1}{2}}\right)^{2}=X$.

Lemma 3.1 Assume that $\alpha \in \mathrm{H}_{n,+} \backslash\{0\}$. Let $D_{\alpha}: \mathrm{H}_{n,++} \rightarrow \mathrm{H}_{n,+, 1}$ be given by

$$
D_{\alpha}(X)=\frac{1}{\operatorname{tr} X^{-1} \alpha} X^{\frac{-1}{2}} \alpha X^{-\frac{1}{2}} \text {. }
$$

If $\alpha \in \mathrm{H}_{n,++}$ then $D_{\alpha}\left(\mathrm{H}_{n,++}\right) \subseteq \mathrm{H}_{n,++, 1}$. Assume that a,b satisfy (3.2). Then

$$
\begin{aligned}
& D_{\alpha}(\mathrm{H}(a, b, 1)) \subseteq \mathrm{H}(c, d, 1), \\
& c=\frac{a \lambda_{n}(\alpha)}{a \lambda_{n}(\alpha)+(n-1) b \lambda_{1}(\alpha)}, d=\frac{b \lambda_{1}(\alpha)}{b \lambda_{1}(\alpha)+(n-1) a \lambda_{n}(\alpha)} .
\end{aligned}
$$

Proof. Clearly, for $X \in \mathrm{H}_{n,++}$ and $\alpha \in \mathrm{H}_{n,+} \backslash\{0\}$ we have that $X^{\frac{-1}{2}} \alpha X^{-\frac{1}{2}} \in$ $\mathrm{H}_{n,+} \backslash\{0\}$. Hence $D_{\alpha}\left(\mathrm{H}_{n,++}\right) \subseteq \mathrm{H}_{n,+, 1}$. Furthermore if $\alpha \in \mathrm{H}_{n,++, 1}$ then $D_{\alpha}\left(\mathrm{H}_{n,++, 1}\right) \subseteq$ $\mathrm{H}_{n,++, 1}$.

Assume that $\alpha \in \mathrm{H}_{n,++, 1}$. Clearly,

$$
\lambda_{1}\left(X^{\frac{-1}{2}} \alpha X^{-\frac{1}{2}}\right)=\left\|X^{\frac{-1}{2}} \alpha X^{-\frac{1}{2}}\right\| \leq\left\|X^{-\frac{1}{2}}\right\|^{2}\|\alpha\|=\frac{\lambda_{1}(\alpha)}{\lambda_{n}(X)} \leq \frac{\lambda_{1}(\alpha)}{a} .
$$

As $\lambda_{n}\left(X^{\frac{-1}{2}} \alpha X^{-\frac{1}{2}}\right)=\frac{1}{\lambda_{1}\left(X^{\frac{1}{2}} \alpha^{-1} X^{\frac{1}{2}}\right)}$ it follows that

$$
\lambda_{n}\left(X^{\frac{-1}{2}} \alpha X^{-\frac{1}{2}}\right) \geq \frac{\lambda_{n}(\alpha)}{\lambda_{1}(X)} \geq \frac{\lambda_{n}(\alpha)}{b}
$$

Observe next that if $0<f \leq x_{n} \leq \cdots \leq x_{1} \leq g$ then

$$
\frac{f}{f+(n-1) g} \leq \frac{x_{n}}{\sum_{i=1}^{n} x_{i}} \leq \ldots \leq \frac{x_{1}}{\sum_{i=1}^{n} x_{i}} \leq \frac{g}{g+(n-1) f} .
$$

Combine the above results to deduce (3.6). The continuity argument yields (3.6) for $\alpha \in \mathrm{H}_{n,+, 1}$.

For a positive integer $k$ let $[k]:=\{1, \ldots, k\}$. Recall that on $\mathbb{C}^{n \times n}$ one has the inner product $\langle X, Y\rangle=\operatorname{tr} X Y^{*}$. For a completely positive operator $Q$ given by (1.1) let:

$$
Q^{\prime}(X)=\sum_{i=1}^{k} A_{i}^{*} X A_{i}, \quad A_{i} \in \mathbb{C}^{n \times n}, i \in[k] .
$$


Then $Q^{\prime}$ is the dual of $Q$. Indeed, $\langle Q(X), Y\rangle=\left\langle X, Q^{\prime}(Y)\right\rangle$. Clearly, $Q^{\prime}$ is completely positive. Thus $Q$ is a quantum channel if and only if $Q^{\prime}\left(I_{n}\right)=I_{n}$. $Q$ is called a unital channel if $Q^{\prime}\left(I_{n}\right)=Q\left(I_{n}\right)=I_{n}$. A unital channel is an analog of a doubly stochastic matrices, and sometimes is called doubly stochastic quantum channel [8].

For $A, B \in \mathrm{H}_{n}$ we denote $A \succeq B, A \succeq B, A \succ B$ if $A-B$ is positive semidefinite, nonzero positive semi-definite, positive definite, respectively. Clearly, any completely operator $Q: \mathbb{C}^{n \times n} \rightarrow \mathbb{C}^{n \times n}$ is order preserving on $\mathrm{H}_{n}$, i.e. $X \succeq Y \Rightarrow$ $Q(X) \succeq Q(Y)$. Hence $Q^{\prime}(X) \succeq 0$ if $X \succeq 0$.

Assume that $X, Y \in \mathrm{H}_{n,+}$ then $X^{\frac{1}{2}} Y X^{\frac{1}{2}} \in \mathrm{H}_{n,+}$. Therefore $\operatorname{tr} X Y=\operatorname{tr} X^{\frac{1}{2}} Y X^{\frac{1}{2}} \geq$ 0 . Hence if $U \preceq V$ it follows that $\operatorname{tr} U Y \leq \operatorname{tr} V Y$.

Lemma 3.2 Assume that $Q: \mathbb{C}^{n \times n}$ is a completely positive operator. Then

$$
a(Q)=a\left(Q^{\prime}\right), \quad b(Q)=b\left(Q^{\prime}\right)
$$

In particular $Q$ is positive if and only if $Q^{\prime}$ is positive.

Proof. From the definition of $a(Q), b(Q)$ given by (3.3) it follows that $a(Q) I_{n} \leq$ $Q(X) \leq b(Q) I_{n}$ for each $X \in \mathrm{H}_{n,+.1}$. From the definition of $a\left(Q^{\prime}\right), b\left(Q^{\prime}\right)$ it follows that there exists $U, V \in \mathrm{H}_{n,+, 1}$ and $\mathbf{u}, \mathbf{v} \in \mathbb{C}^{n},\|\mathbf{u}\|=\|\mathbf{v}\|=1$ such that

$$
a\left(Q^{\prime}\right)=\lambda_{n}\left(Q^{\prime}(U)\right)=\mathbf{u}^{*} Q^{\prime}(U) \mathbf{u}, \quad b\left(Q^{\prime}\right)=\lambda_{1}\left(Q^{\prime}(V)\right)=\mathbf{v}^{*} Q^{\prime}(V) \mathbf{v} .
$$

Hence

$$
\begin{aligned}
& a\left(Q^{\prime}\right)=\operatorname{tr} \mathbf{u} \mathbf{u}^{*} Q^{\prime}(U)=\operatorname{tr} Q\left(\mathbf{u u}^{*}\right) U \geq \operatorname{tr} a(Q) I_{n} U=a(Q), \\
& b\left(Q^{\prime}\right)=\operatorname{tr} \mathbf{v} \mathbf{v} * Q^{\prime}(V)=\operatorname{tr} Q\left(\mathbf{v v}^{*}\right) V \leq \operatorname{tr} b(Q) I_{n} V=b(Q) .
\end{aligned}
$$

As $\left(Q^{\prime}\right)^{\prime}=Q$ it follows that $a(Q)=a\left(\left(Q^{\prime}\right)^{\prime}\right) \geq a\left(Q^{\prime}\right)$ and $b\left(Q^{\prime}\right) \geq b\left(\left(Q^{\prime}\right)^{\prime}\right)=b(Q)$. Hence (3.8) holds. In particular, $a(Q)>0$ if and only if $a\left(Q^{\prime}\right)>0$.

Lemma 3.2 and the arguments of the proof of Lemma 3.1 yield.

Corollary 3.3 Assume that $Q$ is a positive completely positive operator given by (1.1). Let $Q^{\prime}$ be given by (3.7). Denote by $\tilde{Q}: \mathrm{H}_{n,+, 1} \rightarrow \mathrm{H}_{n,++, 1}$ the following nonlinear operator

$$
\tilde{Q}(X)=\frac{1}{\operatorname{tr} Q^{\prime}(X)} Q^{\prime}(X), \quad X \in \mathrm{H}_{n,+, 1}
$$

Then

$$
\begin{aligned}
& \tilde{Q}\left(\mathrm{H}_{n,+, 1}\right) \subseteq \mathrm{H}_{n}(e, f, 1), \\
& \frac{a(Q)}{a(Q)+(n-1) b(Q)} \leq e \leq f \leq \frac{b(Q)}{b(Q)+(n-1) a(Q)} .
\end{aligned}
$$

Assume that $Q$ is a unital quantum channel. In this case $\tilde{Q}=Q^{\prime}$. Lemma 3.2 yields that in (3.10) we can assume that $e=a(Q)$ and $f=b(Q)$. 


\section{Existence of scaling for positive quantum channel}

Theorem 4.1 Let $Q: \mathbb{C}^{n \times n} \rightarrow \mathbb{C}^{n \times n}$ be a positive quantum channel. Assume that $\alpha, \beta \in \mathrm{H}_{n,+, 1}$. Consider the following continuous nonlinear transformation $\Phi_{\alpha, \beta}: \mathrm{H}_{n,+, 1} \rightarrow \mathrm{H}_{n,+, 1}$ :

$$
\Phi_{\alpha, \beta}=D_{\alpha} \circ \tilde{Q} \circ D_{\beta} \circ Q .
$$

Then $\Phi_{\alpha, \beta}$ has a fixed point $U(\alpha, \beta) \in \mathrm{H}_{n,+, 1}$. If $\alpha \in \mathrm{H}_{n,++, 1}$ then $U(\alpha, \beta) \in$ $\mathrm{H}_{n,++, 1}$.

Proof. Let $\Psi_{1}=D_{\beta} \circ Q, \Psi_{2}=\tilde{Q} \circ D_{\beta} \circ Q$. As $Q$ is positive $Q\left(\mathrm{H}_{n,+, 1}\right) \subseteq$ $\mathrm{H}_{n}(a(Q), b(Q), 1)$. Hence $\Psi_{1}$ is continuous on $Q\left(\mathrm{H}_{n,+, 1}\right)$. Similarly, $\Psi_{2}\left(\mathrm{H}_{n,+, 1}\right) \subset$ $\tilde{Q}\left(\mathrm{H}_{n,+, 1}\right) \subseteq \mathrm{H}_{n}(a(Q), b(Q), 1)$. Thus $\Phi_{\alpha, \beta}$ is continuous on $\mathrm{H}_{n,+, 1}$. Brouwer's fixed point theorem yields that $\Phi_{\alpha, \beta}$ has a fixed point $U(\alpha, \beta) \in \mathrm{H}_{n,+, 1}$.

Assume finally that $\alpha \in \mathrm{H}_{n,++, 1}$. Then $D_{\alpha}\left(\mathrm{H}_{n}(a(Q), b(Q), 1)\right) \subset \mathrm{H}_{n,++, 1}$. Hence $U(\alpha, \beta) \in \mathrm{H}_{n,++, 1}$.

Theorem 4.2 Let $Q: \mathbb{C}^{n \times n} \rightarrow \mathbb{C}^{n \times n}$ be a positive quantum channel. Assume that $\alpha, \beta \in \mathrm{H}_{n,++, 1}$. Then each fixed point of $\Phi_{\alpha, \beta}$, given by (4.1), induces a scaling of the quantum channel $Q$ to a quantum channel $R$ satisfying $R(\alpha)=\beta$. Vice versa, each scaling of the quantum channel $Q$ to a quantum channel $R$ satisfying $R(\alpha)=\beta$ induces a fixed point of $\Phi_{\alpha_{1}, \beta_{1}}$, for $\alpha_{1}=O \alpha O^{*}, \beta_{1}=P \beta P^{*}$ for some unitary $O$ and $P$.

Proof. Suppose that $\Phi_{\alpha, \beta}(U)=U$ for some $U \in \mathrm{H}_{n,++1}$. Denote $V=$ $Q(U), W=D_{\beta}(V), Z=\tilde{Q}(W)$. Then $D_{\alpha}(Z)=U$. Observe that $Q^{\prime}(W)=t^{-2} Z$ for some $t>0$. Let $T=t Z^{-\frac{1}{2}}, S=W^{\frac{1}{2}}$. Let $R_{1}: \mathbb{C}^{n \times n} \rightarrow \mathbb{C}^{n \times n}$ be given by $R_{1}(X)=S Q\left(T X T^{*}\right) S^{*}$. Clearly, $R_{1}$ is a scaling of $R$. Furthermore

$$
R_{1}^{\prime}\left(I_{n}\right)=T^{*} Q^{\prime}\left(S^{*} S\right) T=\left(t Z^{-\frac{1}{2}}\right) Q^{\prime}(W)\left(t Z^{-\frac{1}{2}}\right)=\left(t Z^{-\frac{1}{2}}\right)\left(t^{-2} Z\right)\left(t Z^{-\frac{1}{2}}\right)=I_{n} .
$$

Hence $R_{1}$ is a quantum channel.

We now claim that there exists a unitary $O$ such that $R_{1}(\alpha)=O \beta O^{*}$. Indeed,

$$
Q(U)=Q\left(D_{\alpha}(Z)\right)=Q\left(\frac{1}{\operatorname{tr} Z^{-1} \alpha} Z^{-\frac{1}{2}} \alpha Z^{-\frac{1}{2}}\right)=\frac{1}{t^{2} \operatorname{tr} Z^{-1} \alpha} Q\left(T \alpha T^{*}\right)=V .
$$

Hence $R_{1}(\alpha)=(s t)^{2} W^{\frac{1}{2}} V W^{\frac{1}{2}}$ for some $s=\sqrt{\operatorname{tr} Z^{-1} \alpha}$. The equality $W=D_{\beta}(V)$ is equivalent to $W=r^{2} V^{-\frac{1}{2}} \beta V^{-\frac{1}{2}}$ for $r=\frac{1}{\sqrt{\operatorname{tr} V^{-1} \beta}}$. That is

$$
\left(W^{\frac{1}{2}}\right)^{2}=\left(r V^{-\frac{1}{2}} \beta^{\frac{1}{2}}\right)\left(r V^{-\frac{1}{2}} \beta^{\frac{1}{2}}\right)^{*} \Rightarrow\left(r W^{-\frac{1}{2}} V^{-\frac{1}{2}} \beta^{\frac{1}{2}}\right)\left(r W^{-\frac{1}{2}} V^{-\frac{1}{2}} \beta^{\frac{1}{2}}\right)^{*}=I_{n}
$$

Therefore $O:=r W^{-\frac{1}{2}} V^{-\frac{1}{2}} \beta^{\frac{1}{2}}$ is a unitary matrix. Clearly, $V^{\frac{1}{2}} W^{\frac{1}{2}}=r \beta^{\frac{1}{2}} O^{*}$. Finally,

$$
R_{1}(\alpha)=(s t)^{2} W^{\frac{1}{2}} V W^{\frac{1}{2}}=(s t)^{2}\left(W^{\frac{1}{2}} V^{\frac{1}{2}}\right)\left(W^{\frac{1}{2}} V^{\frac{1}{2}}\right)^{*}=(r s t)^{2} O \beta O^{*} .
$$

As $\alpha, \beta \in \mathrm{H}_{n,+, 1}$ and $R_{1}$ is a trace preserving, it follows that $(r s t)^{2}=1$. Hence $R_{1}(\alpha)=O \beta O^{*}$. Define $R(X)=O^{*} R_{1}(X) O$. Clearly, $R$ a scaling of $Q, R$ is a quantum channel, and $R(\alpha)=\beta$. 
Assume now that $R$ is a scaling of the quantum channel $Q$ such that $R$ is a quantum channel, and $R(\alpha)=\beta$. Let $R$ be given by (1.3). Define $W:=s S^{*} S$, where $s=\frac{1}{\operatorname{tr} S^{*} S}$. Let $Z=\tilde{Q}(W)$. As $R$ is a quantum channel we deduce that $Z=t\left(T^{*}\right)^{-1} T^{-1}$, where $t=\frac{1}{\operatorname{tr}\left(T^{*}\right)^{-1} T^{-1}}$. From the above arguments it follows that

$$
Z^{\frac{1}{2}}=t^{\frac{1}{2}}\left(T^{*}\right)^{-1} O^{*}=t^{\frac{1}{2}} O T^{-1}
$$

for some unitary matrix $O$. Let $\alpha_{1}=O \alpha O^{*}$. Then

$$
U=D_{\alpha_{1}}(Z)=r^{-2} T O^{*} \alpha_{1} O T^{*}=r^{-2} T \alpha T^{*}, \quad r^{2}=\operatorname{tr} T \alpha T^{*} .
$$

Then $U=r^{-2} T \alpha T^{*}$. As $R(\alpha)=\beta$ it follows that $V=Q(U)=r^{-2} S^{-1} \beta\left(S^{*}\right)^{-1}$. Hence $V^{\frac{1}{2}}=r^{-1} S^{-1} \beta^{\frac{1}{2}} P^{*}=r^{-1} P \beta^{\frac{1}{2}}\left(S^{*}\right)^{-1}$ for some unitary $P$. Let $\beta_{1}=P \beta P^{*}$. Then

$$
D_{\beta_{1}}(V)=q S^{*} \beta^{-\frac{1}{2}} P^{*} \beta_{1} P \beta^{-\frac{1}{2}} S=q S^{*} S, \quad q=\frac{1}{\operatorname{tr} S^{*} S} .
$$

Therefore $D_{\beta_{1}}(V)=W$, and $U$ is a fixed point of $\Phi_{\alpha_{1}, \beta_{1}}$.

Note that if $Q$ is a quantum channel that satisfies $Q(\alpha)=\beta$, then the scaled channel $R$ given by (1.3), with $S$ and $T$ unitary, is also a quantum channel with $R\left(T^{*} \alpha T\right)=S \beta S^{*}$. This observation explains the second part of Theorem 4.2.

Theorem 4.2 proves the Georgiou-Pavon conjecture [6, Conjecture 1] for two positive definite density matrices:

Conjecture 4.3 (Georgiou-Pavon) Given a positive quantum channel $Q: \mathbb{C}^{n \times n} \rightarrow$ $\mathbb{C}^{n \times n}$ and two density matrices $\rho_{0}, \rho_{T}$ then there exists $\phi_{0}, \phi_{T}, \hat{\phi}_{0}, \hat{\phi}_{T} \in \mathrm{H}_{n,++}$ such that

$$
\begin{aligned}
& Q\left(\phi_{T}\right)=\phi_{0}, \quad Q^{\prime}\left(\hat{\phi}_{0}\right)=\hat{\phi}_{T}, \\
& \rho_{0}=\chi_{0} \hat{\phi}_{0} \chi_{0}^{*}, \quad \rho_{T}=\chi_{T} \hat{\phi}_{T} \chi_{T}^{*}, \\
& \phi_{0}=\chi_{0}^{*} \chi_{0}, \quad \phi_{T}=\chi_{T}^{*} \chi_{T} .
\end{aligned}
$$

Furthermore, $\chi_{0}$ and $\chi_{T}$ can be chosen to be in $\mathrm{H}_{n,+}$.

It is shown in [6] that this conjecture holds for $\rho_{0}=\rho_{T}=\frac{1}{n} I_{n}$, see also [7], and for rank one matrices $\rho_{0}, \rho_{T} \in \mathrm{H}_{n,+, 1}$.

Proof of Georgiou-Pavon conjecture for $\rho_{0}, \rho_{T} \in \mathrm{H}_{n,++.1}$ and nonhermitian $\chi_{0}, \chi_{T}$. Let $\alpha=\rho_{T}$ and $\beta=\rho_{0}$. Theorem 4.1 implies that $\Phi_{\alpha, \beta}$ has a fixed point in $\mathrm{H}_{n,++, 1}$. In the proof of Theorem 4.2 we constructed the quantum channel $R_{1}(X)=S Q\left(T X T^{*}\right) S^{*}$ such that $R_{1}(\alpha)=O \beta O^{*}$ for a corresponding unitary $O$. Furthermore $S, T \in \mathrm{H}_{n,++}$. Thus

$$
Q(T \alpha T)=S^{-1} O \beta O^{*} S^{-1}
$$

Since $R_{1}$ is a quantum channel it follows that $Q^{\prime}\left(S^{2}\right)=T^{-2}$. Choose

$$
\phi_{T}=T \alpha T, \phi_{0}=S^{-1} O \beta O^{*} S^{-1}, \hat{\phi}_{0}=S^{2}, \hat{\phi}_{T}=T^{-2}, \chi_{0}=\beta^{\frac{1}{2}} O^{*} S^{-1}, \chi_{T}=\alpha^{\frac{1}{2}} T \text {. }
$$


Then

$$
\begin{aligned}
& Q\left(\phi_{T}\right)=\phi_{0}, \quad Q^{\prime}\left(\hat{\phi}_{0}\right)=\hat{\phi}_{T}, \\
& \chi_{0}^{*} \chi_{0}=S^{-1} O \beta^{\frac{1}{2}} \beta^{\frac{1}{2}} O^{*} S^{-1}=\phi_{0}, \quad \chi_{T}^{*} \chi_{T}=T \alpha^{\frac{1}{2}} \alpha^{\frac{1}{2}} T=\phi_{T}, \\
& \rho_{0}=\beta=\beta^{\frac{1}{2}} O^{*} S^{-1} S^{2} S^{-1} O \beta^{\frac{1}{2}}=\chi_{0} \hat{\phi}_{0} \chi_{0}^{*}, \quad \rho_{T}=\alpha=\alpha^{\frac{1}{2}} T T^{-2} T \alpha^{\frac{1}{2}}=\chi_{T} \hat{\phi}_{T} \chi_{T}^{*} .
\end{aligned}
$$

We now note that Conjecture 4.3, under the assumption that $\rho_{T}$ and $\rho_{0}$ are positive definite, implies that $Q$ can be scaled to a quantum channel $R$ satisfying $R\left(\rho_{T}\right)=\rho_{0}$. Observe first that (4.3) yields that $\chi_{0}, \chi_{T}$ are invertible. Let

$$
T=\chi_{T}^{*} \rho_{T}^{-\frac{1}{2}}, S=\rho_{0}^{\frac{1}{2}}\left(\chi_{0}^{*}\right)^{-1}, \quad R(X)=S Q\left(T X T^{*}\right) S^{*} .
$$

Then

$$
\begin{aligned}
& R\left(\rho_{T}\right)=S Q\left(T \rho_{T} T^{*}\right) S^{*}=S Q\left(\chi_{T}^{*} \rho_{T}^{-\frac{1}{2}} \rho_{T} \rho_{T}^{-\frac{1}{2}} \chi_{T}\right) S^{*}=S Q\left(\chi_{T}^{*} \chi_{T}\right) S^{*}= \\
& S Q\left(\phi_{T}\right) S^{*}=S \phi_{0} S^{*}=S \chi_{0}^{*} \chi_{0} S^{*}=\rho_{0}^{\frac{1}{2}}\left(\chi_{0}^{*}\right)^{-1} \chi_{0}^{*} \chi_{0} \chi_{0}^{-1} \rho_{0}^{\frac{1}{2}}=\rho_{0}, \\
& R^{\prime}\left(I_{n}\right)=T^{*} Q^{\prime}\left(S^{*} S\right) T=T^{*} Q^{\prime}\left(\chi_{0}^{-1} \rho_{0}\left(\chi_{0}^{*}\right)^{-1}\right)=T^{*} Q^{\prime}\left(\hat{\phi}_{0}\right) T= \\
& T^{*} \hat{\phi}_{T} T^{*}=\rho_{T}^{-\frac{1}{2}} \chi_{T} \hat{\phi}_{T} \chi_{T}^{*} \phi_{T}^{-\frac{1}{2}}=\rho_{T}^{-\frac{1}{2}} \rho_{T} \rho_{T}^{-\frac{1}{2}}=I_{n} .
\end{aligned}
$$

\section{$5 \quad$ Uniqueness of fixed points}

Denote by $\mathbb{P H}_{n,++}$ the space of all rays $t X, X \in \mathrm{H}_{n,++}, t>0$. Clearly, we can identify $\mathbb{P H}_{n,++}$ with $\mathrm{H}_{n,++, 1}$. The Hilbert metric on $\mathbb{P H}_{n,++}$ is given as follows:

$$
\operatorname{dist}(X, Y)=\log \lambda_{1}\left(X Y^{-1}\right)-\log \lambda_{n}\left(X Y^{-1}\right), \quad X, Y \in \mathrm{H}_{n,++} .
$$

To justify that $\operatorname{dist}(X, Y)$ is a metric on $\mathbb{P H}_{n,++}$ we recall the following facts.

$$
\lambda_{1}\left(X Y^{-1}\right)=\lambda_{1}\left(Y^{-\frac{1}{2}} X Y^{-\frac{1}{2}}\right)=\left\|Y^{-\frac{1}{2}} X Y^{-\frac{1}{2}}\right\|, \lambda_{n}\left(X Y^{-1}\right)=\frac{1}{\left\|Y^{\frac{1}{2}} X^{-1} Y^{\frac{1}{2}}\right\|} .
$$

Hence

$$
\begin{aligned}
& \lambda_{1}\left(X Z^{-1}\right)=\lambda_{1}\left(Y^{-\frac{1}{2}} X Y^{-\frac{1}{2}} Y^{\frac{1}{2}} Z^{-1} Y^{\frac{1}{2}}\right) \leq\left\|Y^{-\frac{1}{2}} X Y^{-\frac{1}{2}} Y^{\frac{1}{2}} Z^{-1} Y^{\frac{1}{2}}\right\| \leq \\
& \left\|Y^{-\frac{1}{2}} X Y^{-\frac{1}{2}}\right\|\left\|Y^{\frac{1}{2}} Z^{-1} Y^{\frac{1}{2}}\right\|=\lambda_{1}\left(X Y^{-1}\right) \lambda_{1}\left(Y Z^{-1}\right) .
\end{aligned}
$$

By replacing $X, Y$ and $Z$ by their inverses and using (3.4) we deduce

$$
\lambda_{n}\left(X Z^{-1}\right) \geq \lambda_{n}\left(X Y^{-1}\right) \lambda_{n}\left(Y Z^{-1}\right)
$$

Combine this two inequalities to deduce that

$$
0 \leq \operatorname{dist}(X, Z) \leq \operatorname{dist}(X, Y)+\operatorname{dist}(X, Z) \text { for } X, Y, Z \in \mathrm{H}_{n,++} \text {. }
$$

Suppose that $\lambda_{1}\left(X Y^{-1}\right)=\lambda_{n}\left(X Y^{-1}\right)$. Hence $\lambda_{1}\left(Y^{-\frac{1}{2}} X Y^{-\frac{1}{2}}\right)=\lambda_{n}\left(Y^{-\frac{1}{2}} X Y^{-\frac{1}{2}}\right)$. Therefore $Y^{-\frac{1}{2}} X Y^{-\frac{1}{2}}=t I \Rightarrow X=t Y$ for some $t>0$. 
Assume that $\alpha \in \mathrm{H}_{n,++}$. As the maps $Q, Q^{\prime}, \tilde{Q}, D_{\alpha}$ are homogeneous maps of degrees $1,1,0,0$ it follows that the maps $Q, Q^{\prime}, \tilde{Q}, D_{\alpha}: \mathrm{H}_{n,++} \rightarrow \mathrm{H}_{n,++}$ induce the corresponding maps from $\mathbb{P H}_{n,++}$ to itself. By abusing notation we denote these maps as $Q, Q^{\prime}, \tilde{Q}, D_{\alpha}: \mathbb{P H}_{n,++} \rightarrow \mathbb{P H}_{n,++}$ and no ambiguity will arise. Clearly, the maps $Q^{\prime}$ and $\tilde{Q}$ from $\mathbb{P H}_{n,++}$ to itself are identical. Furthermore, we identify the maps $Q, \tilde{Q}, D_{\alpha}: \mathbb{P H}_{n,++} \rightarrow \mathbb{P H}_{n,++}$ with the corresponding maps $Q, \tilde{Q}, D_{\alpha}$ : $\mathrm{H}_{n,++, 1} \rightarrow \mathbb{P H}_{n,++, 1}$. We also will view $\mathrm{H}_{n,++, 1}$ as metric space with respect the the Hilbert metric.

Theorem 5.1 Let $Q$ be a positive quantum channel given by (1.1). Then

1. The maps $Q$ and $\tilde{Q}$ are strict contractions on $\mathbb{P H}_{n,++}$ :

$$
\begin{array}{r}
\operatorname{dist}(Q(X), Q(Y) \leq \kappa \operatorname{dist}(X, Y), \operatorname{dist}(\tilde{Q}(X), \tilde{Q}(Y)) \leq \kappa \operatorname{dist}(X, Y), \\
\kappa=\frac{b(Q)-a(Q)}{b(Q)+a(Q)} .
\end{array}
$$

2. The map $D_{t I_{n}}: \mathrm{H}_{n,++} \rightarrow \mathrm{H}_{n,++}$ preserves the Hilbert metric on $\mathbb{P H}_{n,++}$ for $t>0$.

3. The map $\Theta=\Phi_{\frac{1}{n} I_{n}, \frac{1}{n} I_{n}}: \mathrm{H}_{n,++, 1} \rightarrow \mathrm{H}_{n,++, 1}$ is a contraction with respect the Hilbert metric. The contraction constant is bounded by $\kappa^{2}$.

4. $\Theta$ has a unique fixed point $F \in \mathrm{H}_{n,+, 1}$ which lies in the interior of $\mathrm{H}_{n,+, 1}$. For each $X \in \mathrm{H}_{n,+, 1}$ the iterations $\Theta^{\circ m}(X), m \in \mathbb{N}$ converge $F$.

5. There exist open balls $B_{1}, B_{2} \subset \mathrm{H}_{n,++, 1}$, in the Frobenius norm, centered at $\frac{1}{n} I_{n}, F$, respectively, with positive radii $\varepsilon_{1}, \varepsilon_{2}$ respectively, with the following properties. Assume that $\alpha, \beta \in B_{1}$. Then $\Phi_{\alpha, \beta}$ has a unique fixed point $U=U(\alpha, \beta)$ which lies in $B_{2}$. Furthermore, for each $X \in B_{2}$ the iterations $\Theta^{\circ m}(X), m \in \mathbb{N}$ converge $U(\alpha, \beta)$.

Proof. 1. We first show the first inequality of (5.3). Recall that the map $Q: \mathrm{H}_{n,+} \rightarrow \mathrm{H}_{n,+}$ is linear. Hence we can apply Birkhoff's theorem [1], which gives an upper bound on the strict contraction of $Q$ on $\mathbb{P H}_{n,++}$. Namely, let

$$
\Delta(Q):=\max \left\{\operatorname{dist}(Q(X), Q(Y)), X, Y \in \mathrm{H}_{n,+, 1}\right\} .
$$

Then

$$
\operatorname{dist}(Q(X), Q(Y)) \leq \tanh \left(\frac{1}{4} \Delta(Q)\right) \operatorname{dist}(X, Y) \text { for all } X, Y \in \mathrm{H}_{n,++} .
$$

As $a(Q) I_{n} \preceq Q(X), Q(Y) \preceq Q(b) I_{n}$ it follows that $\frac{a(Q)}{b(Q)} Q(Y) \preceq Q(X) \preceq \frac{b(Q)}{a(Q)} Q(Y)$. Hence $\operatorname{dist}(Q(X), Q(Y)) \leq 2 \log \frac{b(Q)}{a(Q)}$. Therefore $\Delta(Q) \leq 2 \log \frac{b(Q)}{a(Q)}$. Note that

$$
\tanh \left(\frac{1}{4} \Delta(Q)\right) \leq \tanh \left(\frac{1}{2} \log \frac{b(Q)}{a(Q)}\right)=\frac{\sqrt{\frac{b(Q)}{a(Q)}}-\sqrt{\frac{a(Q)}{b(Q)}}}{\sqrt{\frac{b(Q)}{a(Q)}}+\sqrt{\frac{a(Q)}{b(Q)}}}=\frac{\frac{b(Q)}{a(Q)}-1}{\frac{b(Q)}{a(Q)}+1}=\frac{b(Q)-a(Q)}{b(Q)+a(Q)}
$$

This shows the first part of the inequality (5.3). 
Consider $\tilde{Q}: \mathbb{P H}_{n,++} \rightarrow \mathbb{P H}_{n,++}$. Clearly, $\operatorname{dist}(\tilde{Q}(X), \tilde{Q}(Y))=\operatorname{dist}\left(Q^{\prime}(X), Q^{\prime}(Y)\right)$. Hence, it is enough to estimate the contraction of $Q^{\prime}$. Apply Lemma 3.2 and the above arguments to deduce the second part of the inequality (5.3).

2. Let $\Omega: \mathrm{H}_{n,++} \rightarrow \mathrm{H}_{n,++}$ be given by $\Omega(X)=X^{-1}$. Clearly, $\operatorname{dist}\left(D_{t I_{n}}(X), D_{t I_{n}}(Y)\right)=$ $\operatorname{dist}(\Omega(X), \Omega(Y))$ for $X, Y \in \mathrm{H}_{n,++}$. Observe that

$$
\lambda_{1}\left(\Omega(X) \Omega(Y)^{-1}\right)=\lambda_{n}\left(X Y^{-1}\right)^{-1}, \lambda_{n}\left(\Omega(X) \Omega(Y)^{-1}\right)=\lambda_{1}\left(X Y^{-1}\right)^{-1}
$$

for $X, Y \in \mathrm{H}_{n,++}$. Hence $D_{t I_{n}}$ preserves the metric on $\mathrm{H}_{n,++}$ for $t>0$. 3 follows straightforward from 2 and 3.

4. As $\Theta\left(\mathrm{H}_{n,+, 1}\right) \subset \mathrm{H}_{n,++, 1}$ and $\Theta$ is a contraction on $\mathrm{H}_{n,++, 1}$, Banach Fixed Point Theorem yields that $F \in \mathrm{H}_{n,++, 1}$ is a unique fixed point of $\Theta$. Furthermore, for each $X \in \mathrm{H}_{n,+, 1}$ the iterations $\Theta^{\circ m}(X), m \in \mathbb{N}$ converge $F$.

5. Denote by $\mathcal{S}_{n}, \mathcal{A}_{n} \subset \mathbb{R}^{n \times n}$ the space of $n \times n$ real symmetric and real skew symmetric matrices. Note that $\operatorname{dim} \mathcal{S}_{n}=\frac{n(n+1)}{2}$ and $\operatorname{dim} \mathcal{A}_{n}=\frac{n(n-1)}{2}$. We now consider $X \in \mathrm{H}_{n}$ as of the form $X=X_{\Re}+\mathbf{i} X_{\Im}$, where $X_{\Re} \in \mathcal{S}_{n}, X_{\Im} \in \mathcal{A}_{n}$. That is we view $\mathrm{H}_{n}$ as the space $\mathbb{R}^{n^{2}}$, where the $n^{2}$ variables are the real entries of the upper diagonal and strict upper diagonal parts of $X_{\Re}$ and $X_{\Im}$ respectively. An open ball of radius $r>0$ centered at $X_{0} \in \mathrm{H}_{n}$ is $B\left(X_{0}, r\right)=\left\{X_{0}+W, W \in \mathrm{H}_{n}, \operatorname{tr} W^{2}<r^{2}\right\}$. Thus $\mathrm{H}_{n,++}$ is a domain in $\mathrm{H}_{n}$. A map $\Lambda: \mathrm{H}_{n,++} \rightarrow \mathrm{H}_{n,++}$ is called real analytic if the following conditions holds. Let $X_{0} \in \mathrm{H}_{n,++}$ be given. Then there exists an open ball centered at $X_{0}$ of radius $\varepsilon=\varepsilon\left(X_{0}, \Lambda\right)$, which is contained in $\mathrm{H}_{n,++}$, such that the following conditions hold. For $X=X_{0}+W$ in this ball the real and the imaginary parts of the entries of $\Lambda\left(X_{0}+W\right)$ are given by convergent power series in the real and complex parts of the entries of $W$. Similar definition applies for analyticity of the map $\Lambda_{1}: \mathrm{H}_{n,++, 1} \rightarrow \mathrm{H}_{n,++, 1}$.

Clearly, the maps $Q, Q^{\prime}: \mathrm{H}_{n,++} \rightarrow \mathrm{H}_{n,++}$ are linear in $n^{2}$ real variables of $X \in \mathrm{H}_{n,++}$. Hence these maps are analytic. It is straightforward to see that the map $\tilde{Q}: \mathrm{H}_{n,++} \rightarrow \mathrm{H}_{n,++, 1} \subset \mathrm{H}_{n,++}$ is analytic. Use the formula for $\Omega(X)$ in terms of the adjoint matrix of $X$, i.e., $\Omega(X)=\frac{1}{\operatorname{det} X} \operatorname{adj} X$, to deduce that $\Omega$ is analytic on $\mathrm{H}_{n,++}$.

Let $\Gamma: \mathrm{H}_{n,++} \rightarrow \mathrm{H}_{n,++}$ be given by $\Gamma(X)=X^{\frac{1}{2}}$. Then $\Gamma$ is analytic on $\mathrm{H}_{n,++}$. This fact can be easily deduced from the Cauchy integral formula $[4, \S 3.4]$. Indeed, let $\mathbb{C}_{+}=\{z \in \mathbb{C}, \Im z>0\}$ be the right half complex plane. Let $f: \mathbb{C}_{+} \rightarrow \mathbb{C}_{+}$be the analytic function $f(z)=\sqrt{z},(\sqrt{1}=1)$. Assume that $X_{0} \in \mathrm{H}_{n,++}$ is given. Let $S$ be the circle centered at $\frac{\lambda_{1}\left(X_{0}\right)+\lambda_{n}\left(X_{0}\right)}{2}$ with radius $\frac{\lambda_{1}\left(X_{0}\right)}{2}$. Assume that $\varepsilon>0$ is small enough so that $\lambda_{n}\left(X_{0}+W\right)>\frac{\lambda_{n}\left(X_{0}\right)}{2}$ for each $W \in B(0, \varepsilon)$. Then

$$
\Gamma\left(X_{0}+W\right)=\frac{1}{2 \pi \mathbf{i}} \int_{S} f(z)\left(z I-\left(X_{0}+W\right)\right)^{-1} d z .
$$

Express $\left(z I-\left(X_{0}+W\right)\right)^{-1}$ in terms of the adjoint of $\left(z I-\left(X_{0}+W\right)\right)$ to deduce the analyticity of $\Gamma$.

Hence the map $D_{\alpha}: \mathrm{H}_{n,++} \rightarrow \mathrm{H}_{n,++1}$ is analytic for $\alpha \in \mathrm{H}_{n,++}$. Let $\Phi_{\alpha, \beta}$ be the map given by (4.1). Then $\Phi_{\alpha, \beta}: \mathrm{H}_{n,++, 1} \rightarrow \mathrm{H}_{n,++, 1}$ is analytic. In particular, $\Theta$ is analytic on $\mathrm{H}_{n,++, 1}$. We now consider $\Theta$ in the neighborhood of the fixed point $F \in \mathrm{H}_{n,++, 1}$. Denote by $\mathrm{H}_{n, 0}=\left\{W \in \mathrm{H}_{n}, \operatorname{tr} W=0\right\}$. Then the Jacobian of $\Theta \mid \mathrm{H}_{n,++, 1}$ at $F$ is a linear map $P: \mathrm{H}_{n, 0} \rightarrow \mathrm{H}_{n, 0}$. That is

$$
\Theta(F+W)=F+P(W)+\text { higher order terms. }
$$


Denote by $\rho(P)$ the spectral radius $P$. We claim that $\rho(P)<1$. By considering a scaled quantum channel, we may assume without loss of generality that $Q$ is a unital channel, i.e., $Q\left(\frac{1}{n} I_{n}\right)=\frac{1}{n} I_{n}$. Hence $F=\frac{1}{n} I_{n}$. Assume that $W \in \mathrm{H}_{n, 0}$ and consider $F(t)=\frac{1}{n} I+t W$. Then

$$
\begin{aligned}
& Q(F(t))=\frac{1}{n} I+t Q(W), D_{\frac{1}{n} I_{n}}\left(\frac{1}{n} I+t Q(W)\right)=\frac{1}{n} I_{n}-t Q(W)+O\left(t^{2}\right), \\
& \left.\tilde{Q}\left(\frac{1}{n}-t Q(W)+O\left(t^{2}\right)\right)=\frac{1}{n} I_{n}-t\left(Q^{\prime} \circ Q\right)(W)\right)+O\left(t^{2}\right), \\
& D_{\frac{1}{n} I_{n}}\left(\frac{1}{n} I-t\left(Q^{\prime} \circ Q\right)(W)+O\left(t^{2}\right)\right)=\frac{1}{n} I_{n}+t\left(Q^{\prime} \circ Q\right)(W)+O\left(t^{2}\right) .
\end{aligned}
$$

Therefore $P=Q^{\prime} \circ Q$ is a selfadjoint linear operator, which is positive semidefinite with respect to the standard inner product on $\mathrm{H}_{n, 0}$. That is, all eigenvalues of $P$ are nonnegative. We claim that $Q$ and $Q^{\prime}$ are strict contraction on $\mathrm{H}_{n, 0}$. Indeed $Q\left(\mathrm{H}_{n,+, 1}\right) \subset \mathrm{H}_{n}(a(Q), b(Q), 1)$ and $Q\left(\frac{1}{n} I_{n}\right)=\frac{1}{n} I_{n}$. Hence any ball in $\mathrm{H}_{n,+, 1}$ centered at $\frac{1}{n} I_{n}$ is mapped to the interior of this ball. Similarly, for $Q^{\prime}$. Hence $P$ is a positive semi-define matrix with $\|P\|=\rho(P)<1$. In particular $P$ is a contraction on $\mathrm{H}_{n, 0}$ with respect to the Frobenius norm.

Consider now $\Phi_{\alpha, \beta}$ as the family of maps depending on parameters $\alpha, \beta$. That is, $\Phi_{\alpha, \beta}$ is given by $(X, \alpha, \beta) \mapsto \Phi_{\alpha, \beta}(X)$ for $(X, \alpha, \beta) \in \mathrm{H}_{n,++}^{3}$. The arguments above show that $\Phi_{\alpha, \beta}$ is analytic on $\mathrm{H}_{n,++}^{3}$. Hence, in the neighborhood of $\left(\frac{1}{n} I_{n}, \frac{1}{n} I_{n}, \frac{1}{n} I_{n}\right)$ in $\mathrm{H}_{n,++, 1}^{3}$ we have:

$$
\begin{aligned}
& \Phi_{\frac{1}{n} I_{n}+\alpha_{1}, \frac{1}{n} I_{n}+\beta_{1}}\left(\frac{1}{n} I_{n}+W\right)= \\
& \frac{1}{n} I_{n}+W_{1}\left(\alpha_{1}, \beta_{1}\right)+\left(P(W)+E_{1}\left(\alpha_{1}, \beta_{1}\right)(W)\right)+E_{2}\left(W, \alpha_{1}, \beta_{1}\right), \\
& \left\|W_{1}\left(\alpha_{1}, \beta_{1}\right)\right\|_{F} \leq K\left(\left\|\alpha_{1}\right\|_{F}+\left\|\beta_{1}\right\|_{F}\right),\left\|E_{1}\left(\alpha_{1}, \beta_{1}\right)\right\| \leq K\left(\left\|\alpha_{1}\right\|_{F}+\left\|\beta_{1}\right\|_{F}\right), \\
& E_{2}\left(0, \alpha_{1}, \beta_{1}\right)=0,\left\|E_{2}\left(W_{1}, \alpha_{1}, \beta_{1}\right)-E_{2}\left(W_{2}, \alpha_{1}, \beta_{1}\right)\right\|_{F} \leq \\
& K\left\|W_{1}-W_{2}\right\|_{F}\left(\left\|W_{1}\right\|_{F}+\left\|W_{2}\right\|_{F}\right)\left(1+\left\|\alpha_{1}\right\|_{F}+\left\|\beta_{1}\right\|_{F}\right) .
\end{aligned}
$$

Note that $W_{1}\left(\alpha_{1}, \beta_{1}\right), E_{2}\left(W, \alpha_{1}, \beta_{1}\right) \in \mathrm{H}_{n, 0}$ and $E_{1}\left(\alpha_{1}, \beta_{1}\right): \mathrm{H}_{n, 0} \rightarrow \mathrm{H}_{n, 0}$ is a linear operator for each $\alpha_{1}, \beta_{1}$ in some open ball of radius $r$ centered at $0 \in \mathrm{H}_{n, 0}$. Furthermore, we assume that the above inequalities hold for $\left\|\alpha_{1}\right\|_{F},\left\|\beta_{1}\right\|_{F},\|W\|,\left\|W_{1}\right\|,\left\|W_{2}\right\|<$ $r$. Assume that $\|P\|<1-2 \eta$, for some $\eta>0$, and $t \in(0, r)$. Suppose that $\left\|\alpha_{1}\right\|,\left\|\beta_{1}\right\|<\frac{t^{2}}{2}$ and $\|W\|_{F}<t$. Then

$$
\left\|\Phi_{\frac{1}{n} I_{n}+\alpha_{1}, \frac{1}{n} I_{n}+\beta_{1}}\left(\frac{1}{n} I_{n}+W\right)-\frac{1}{n} I_{n}\right\|<K t^{2}+\left(1-2 \eta+K t^{2}\right) t+K t^{2}\left(1+t^{2}\right) .
$$

Hence there exists $\varepsilon \in(0, \min (r, 1))$ such that

$$
2 K\left(\varepsilon+\varepsilon^{2}+\varepsilon^{3}\right)<\eta .
$$

In particular

$$
K \varepsilon^{2}+\left(1-2 \eta+K \varepsilon^{2}\right) \varepsilon+K \varepsilon^{2}\left(1+\varepsilon^{2}\right)=\left(1-2 \eta+K\left(2 \varepsilon+\varepsilon^{2}+\varepsilon^{3}\right)\right) \varepsilon<(1-\eta) \varepsilon .
$$

Therefore

$$
\Phi_{\alpha, \beta}\left(B\left(\frac{1}{n} I_{n}, \varepsilon\right)\right) \subset B\left(\frac{1}{n} I_{n}, \varepsilon\right) \text { for } \alpha, \beta \in B\left(\frac{1}{n} I_{n}, \frac{\varepsilon^{2}}{2}\right) .
$$


Assume that $W_{1}, W_{2} \in B(0, \varepsilon)$. Then

$$
\begin{aligned}
& \left\|\Phi_{\alpha, \beta}\left(\frac{1}{n} I_{n}+W_{1}\right)-\Phi_{\alpha, \beta}\left(\frac{1}{n} I_{n}+W_{2}\right)\right\|_{F} \leq \\
& \left(1-2 \eta+K \varepsilon^{2}\right)\left\|W_{1}-W_{2}\right\|+2 K \varepsilon\left(1+\varepsilon^{2}\right)\left\|W_{1}-W_{2}\right\|= \\
& \left(1-2 \eta+K\left(2 \varepsilon+\varepsilon^{2}+2 \varepsilon^{3}\right)\right)\left\|W_{1}-W_{2}<(1-\eta)\right\| W_{1}-W_{2} \| .
\end{aligned}
$$

That is, for fixed $\alpha, \beta \in B\left(\frac{1}{n} I_{n}, \frac{\varepsilon^{2}}{2}\right)$ and $X \in B_{2}=B\left(\frac{1}{n} I_{n}, \varepsilon\right)$ the map $\Phi_{\alpha, \beta}$ is a contraction with respect to the Frobenius norm. Hence $\Phi_{\alpha, \beta}$ has a unique fixed point $U=U(\alpha, \beta)$ in $B_{2}$. Furthermore, for each $X \in B_{2}$ the iterations $\Theta^{\circ m}(X), m \in \mathbb{N}$ converge to $U$.

It is left to show that there exists $\varepsilon_{1} \in\left(0, \frac{\varepsilon^{2}}{2}\right)$ such that for $\alpha, \beta \in B\left(\frac{1}{n} I_{n}, \varepsilon_{1}\right)$ the map $\Phi_{\alpha, \beta}$ have a unique fixed point which is $U(\alpha, \beta)$. Since $\Theta$ has a unique fixed point $\frac{1}{n} I_{n}$ the continuity argument yields that there exists $\varepsilon_{1} \in\left(0, \frac{\varepsilon^{2}}{2}\right)$ such that for each $\alpha, \beta \in B_{1}=B\left(\frac{1}{n} I_{n}, \varepsilon_{1}\right)$ all fixed points of $\Phi_{\alpha, \beta}$ lie in $B_{2}$. Our previous results show that in $B_{2}$ the map $\Phi_{\alpha, \beta}$ has a unique fixed point $U(\alpha, \beta)$.

It is an open problem to prove or give a counterexample that $\Phi_{\alpha, \beta}$ has a unique fixed point in $\mathrm{H}_{n,++, 1}$ for each $\alpha, \beta \in \mathrm{H}_{n,++, 1}$. See [6] for numerical simulations.

\section{References}

[1] G. Birkhoff, Extensions of Jentzsch's theorem, Trans. Amer. Math. Soc. 85 (1957), 219-227.

[2] R.A. Brualdi, Convex sets of nonnegative matrices, Canad. J. Math 20 (1968), 144-157.

[3] R.A. Brualdi, S.V. Parter and H. Schneider, The diagonal equivalence of a nonnegative matrix to a stochastic matrix, J. Math. Anal. Appl.16 (1966), $31-50$.

[4] S. Friedland, Matrices: Algebra, Analysis and Applications, World Scientific, 596 pp., 2015, Singapore.

[5] S. Friedland and S. Karlin, Some inequalities for the spectral radius of nonnegative matrices and applications, (with S. Karlin), Duke Math. J. 42 (1975), 459-490.

[6] T.T. Georgiou and M. Pavon, Positive contraction mappings for classical and quantum Schrödinger systems, J. Math. Physics, 56, 033301, (2015).

[7] L Gurvits, Classical complexity and quantum entanglement, J. Computer and System Sciences, 69 (2004), 448-484.

[8] C.B. Mendl and M.M. Wolf, Unital quantum channels - convex structure and revivals of Birkhoffs theorem, Commun. Math. Phys. 289 (2009), 10571086 . 
[9] M.V. Menon, Matrix links, an extremisation problem and the reduction of a nonnegative matrix to one with with prescribed row and column sums, Canad. J. Math 20 (1968), 225-232.

[10] J. Milnor, Topology from the differentiable viewpoint, Princeton Landmarks in Mathematics. Princeton, N.J., Princeton University Press, 1997.

[11] H. Perfect and L. Mirsky, The distribution of positive elements in doubly stochastic matrices, J. London Math. Soc. 40 (1965), 688-698.

[12] E. Schrödinger, Über die Umkehrung der Naturgesetze, Sitzungs- berichte der Preuss Akad. Wissen. Berlin, Phys. Math. Klasse, (1931), 144-153.

[13] E. Schrödinger, Sur la théorie relativiste de l'électron et l'interprétation de la mécanique quantique, Ann. Inst. H. Poincaré 2, 2 (1932), 269-310.

[14] R. Sinkhorn, A relationship between arbitrary positive matrices and doubly stochastic matrices, Ann. Math. Statist. 35 (1964), 876-879.

[15] R. Sinkhorn and P. Knopp, Concerning nonnegative matrices and doubly stochastic matrices, Pac. J. Math. 21 (1967), 343-348. 